\title{
Enhancement of cognitive and neural functions through complex reasoning training: evidence from normal and clinical populations
}

\author{
Sandra B. Chapman ${ }^{1 *}$ and Raksha A. Mudar ${ }^{2}$ \\ ${ }^{1}$ Center for BrainHealth ${ }^{\circledR}$, The University of Texas at Dallas, Dallas, TX, USA \\ ${ }^{2}$ Department of Speech and Hearing Science, University of Illinois at Urbana-Champaign, Champaign, IL, USA
}

Edited by:

Mikhail Lebedev, Duke University, USA

\section{Reviewed by:}

Jitendra Sharma, Massachusetts Institute of Technology, USA Aaron P. Blaisdell, University of California, Los Angeles, USA

\section{*Correspondence:}

Sandra B. Chapman, Center for BrainHealth ${ }^{\circledR}$, The University of Texas at Dallas, 2200 W

Mockingbird Ln, Dallas, TX, 75235 USA

e-mail:schapman@utdallas.edu
Public awareness of cognitive health is fairly recent compared to physical health. Growing evidence suggests that cognitive training offers promise in augmenting cognitive brain performance in normal and clinical populations. Targeting higher-order cognitive functions, such as reasoning in particular, may promote generalized cognitive changes necessary for supporting the complexities of daily life. This data-driven perspective highlights cognitive and brain changes measured in randomized clinical trials that trained gist reasoning strategies in populations ranging from teenagers to healthy older adults, individuals with brain injury to those at-risk for Alzheimer's disease. The evidence presented across studies support the potential for Gist reasoning training to strengthen cognitive performance in trained and untrained domains and to engage more efficient communication across widespread neural networks that support higher-order cognition. The meaningful benefits of Gist training provide compelling motivation to examine optimal dose for sustained benefits as well as to explore additive benefits of meditation, physical exercise, and/or improved sleep in future studies.

Keywords: cognitive training, gist reasoning, cognition, neural, brain plasticity

\section{INTRODUCTION}

Five decades of research have shown that the brain is modifiable in the context of stimulating cognitive experiences and in response to cognitive training (e.g., Bennett et al., 1964; Schooler, 1984; Schooler et al., 1999). Cognitive training involves guided practice on tasks targeting specific cognitive functions (e.g., working memory, attention) or specific cognitive strategies (Clare and Woods, 2004; Martin et al., 2011). In general, evidence from most cognitive training studies reveal improvements in performance, especially in cognitive functions directly targeted by training (Nyberg et al., 2003; Dahlin et al., 2008; Jaeggi et al., 2008; Valenzuela et al., 2008; Carlson et al., 2009; Zelinski, 2009; Anguera et al., 2013; Chapman et al., 2013). However, much remains to be learned about the kinds of training programs that provide meaningful changes beyond the specific skills trained. From a public health perspective, cognitive training will be deemed useful if the training has generalized benefits and builds cognitive capacities to support performance in day-to-day tasks (Anand et al., 2011).

Concerted efforts are being directed toward exploring the realities of augmenting cognitive performance. Memory training has been a key focus of cognitive training programs motivated by extant evidence that declines in memory commonly occur with normal aging and brain diseases/injuries (e.g., Papp et al., 2009; Gates et al., 2011). Memory training mostly yields short-term improvements in memory; however, benefits do not generalize/transfer to other cognitive functions (Papp et al., 2009; Valenzuela and Sachdev, 2009; Martin et al., 2011; Tardif and Simard, 2011; Reijnders et al., 2012; Teixeira et al., 2012; Thompson et al., 2013), nor do they produce long-term benefits (Rebok et al., 2014). Emerging evidence suggests that reasoning training has widespread and lasting benefits that may guard against and restore cognitive losses in aging and/or disease (Willis et al., 2006; Anand et al., 2011; Chapman et al., 2013; Rebok et al., 2014). For instance, in one of the largest randomized cognitive training trials to date, i.e., the Advanced Cognitive Training for Independent and Vital Elderly (ACTIVE) trial, participants who underwent reasoning training (i.e., focused on solving problems related to serial patterns and sequences) showed less decline in self-reported Independent Activities of Daily Living over a 10 -year period compared to the memory training group (i.e., strategies to improve verbal episodic memory) (Rebok et al., 2014).

A growing body of evidence suggests that advanced reasoning engages gist-based processing (Reyna and Brainerd, 1995, 2011; Reyna and Lloyd, 2006). In the current paper, we present a perspective on a particular type of reasoning training that targets gist-based reasoning abilities. Gist reasoning is defined as the ability to synthesize and create abstract meanings from the literal content/information, a skill essential for academic, occupational, and functional competence. The primary objective of strategydriven Gist reasoning training is to improve the ability to abstract generalized meanings from complex information and to incorporate these strategies into everyday tasks. Gist training is informed by cognitive theories of discourse meaning structure (Van Dijk et al., 1983) and information processing (Reyna and Brainerd, 
1995), specifically van Dijk and Kintsch's macrostructure/global meanings and Reyna and Brainerd's gist representation. In this perspective paper, we provide a general framework of Gist reasoning training and highlight converging findings from Gist reasoning training studies across adolescent and adult populations. This novel strategy-based approach to cognitive training may provide insights and future directions to guide testing and development of training protocols that have ecological validity/real life application.

\section{GENERAL OVERVIEW OF COGNITIVE TRAINING PROTOCOLS}

In the distinct studies summarized below, we examined the potential for Gist reasoning training to improve cognitive performance as compared to control protocol/s (active control/wait-list control) (see Table 1) using a pseudo-randomized control design. We present data from studies on two groups of adolescents [i.e., typically developing eighth graders; youth with traumatic brain injury (TBI)] and three adult populations (i.e., adults with TBI, adults with early mild cognitive impairment, and cognitive healthy adults). Overall, the length of the training period across studies was short-term, ranging from 8 to 12 sessions delivered over one to two months in 45 to $60 \mathrm{~min}$. duration, with each study protocol being identical for all participants within the trial. When an active control protocol was used (Memory strategy or New learning), it was comparable to the Gist reasoning training in length, complexity, and active group discussion/engagement.

\section{GIST REASONING TRAINING}

The Gist reasoning training (also referred to as gist training) is a strategy- rather than content-based program. The protocol entails three core strategies: strategic attention, integrated reasoning, and innovation summarized in Table 1, delineated in a training manual, and defined in more detail elsewhere (e.g., Gamino et al., 2010; Vas et al., 2011; Chapman et al., 2012; Chapman and Mudar, 2013). The strategies facilitate cognitive control and depth of encoding to facilitate knowledge acquisition and creation. The strategy instruction is hierarchical and dynamically interdependent, with each strategy building on previous strategies, and involves practice that encourages integrating all steps when tackling mental activities both inside and outside of training.

\section{EVIDENCE OF GAINS FROM GIST TRAINING vs. CONTROL CONDITIONS COGNITIVE TRAINING IN YOUTH (GAMINO ET AL., 2010; MOTES ET AL., 2014; COOK ET AL., UNDER REVIEW) Typically developing adolescents}

In this study, middle-school students (8th graders) were randomly assigned to one of three training protocols, either the Gist reasoning, or one of two control groups, i.e., the Memory training or the New learning (Gamino et al., 2010). The trainings were delivered over 9 class periods lasting 45 min over four weeks. These students were from lower SES backgrounds (92\% living in poverty). The three groups were comparable in age, gender, memory, and cognitive abilities at baseline. Outcomes comparing preto post- training performances were scored by researchers blinded to individuals and group identity.
Table 1 | Brief description of experimental and control training protocols.

\begin{tabular}{ll}
\hline Training & Description \\
\hline EXPERIMENTAL TRAINING & \\
Gist Reasoning Training (Gamino & Hierarchical Strategies \\
et al., 2010; Anand et al., 2011; & Strategic Attention: Consciously \\
Vas et al., 2011; Chapman et al., & blocking/inhibiting distractions and \\
2013; Mudar et al., 2013; Motes & irrelevant/less relevant information \\
et al., 2014; Cook et al., under & Integrated Reasoning: Binding \\
review) & explicit facts with world knowledge \\
& to construct generalized/abstracted \\
& meanings \\
& Innovation: Deriving multiple \\
& interpretations and generalized \\
& applications beyond the concrete \\
& content reflecting fluency and \\
& fluidity of thinking
\end{tabular}

\section{CONTROL TRAINING}

Memory Strategy Training

(Gamino et al., 2010; Cook et al., under review)

New Learning Training (Vas et al., 2011; Mudar et al., 2013)

(Gamino et al., 2010)

Wait-list controls (Chapman et al., 2013; Motes et al., 2014)

Results revealed that gist-trained students improved ability to abstract novel meanings from lengthy classroom-type texts. Additionally, these gist-trained students showed significant improvement in memory for facts, a skill not targeted in training. We found a significant relation between gains in ability to abstract meanings and a real life school measure, the Texas Assessment of Knowledge and Skills (TAKS) reading testing "Applying Critical Thinking Skills." In contrast, students in the Memory training showed improvements only in memory for isolated facts with no gains in abstracting meanings. The New learning group did not show any significant gains. These findings suggest that gist reasoning strategies may have a broader impact on learning that improves deeper understanding of information encountered beyond shallow learning of isolated facts.

In a subsequent study Motes et al. (2014) examined the effects of Gist training on neural mechanisms related to inhibitory 
control using electroencephalograph (EEG). Participants in the Gist training group vs. a Wait-list control group completed three visual go/no-go tasks that involved varying levels of semantic categorization (basic to more abstract superordinate categorization) both before and after training or a comparable duration in the case of the controls. The findings revealed that participants in the Gist group showed significant improvement in inhibition (i.e., ability to withhold behavioral responses on no-go trials) following training unlike the control group across basic and superordinate categorization tasks. Furthermore, those in the Gist trained group showed EEG changes suggestive of improved processing efficiency, as reflected by significant reduction in P3 no-go amplitude post-training compared to pre-training. No such differences were observed in the Wait-list controls. Overall, both the behavioral findings and the electrophysiological data across these two studies suggest that Gist training appears to enhance inhibitory responses both at the behavioral and neural level in typically developing middle school children. However, these findings have to be further validated using additional procedures (e.g., positive and negative patterning) in future studies.

\section{Adolescents with TBI}

The benefits of Gist training vs. Memory training in adolescents with TBI (ages 12-20 years) at chronic stages post injury ( $>6$ months) was evaluated in a recent study (Cook et al., under review). Participants received one-on-one training delivered in eight sessions of $45 \mathrm{~min}$ duration over a four week period. The text materials for the Memory training were largely the same content as used in the Gist protocol. The findings revealed that the Gist-trained group significantly improved in their ability to abstract/synthesize meanings as compared to the Memorytrained group. The Gist-trained group also showed enhanced performance on cognitive measures for memory for facts, working memory (Digit Span backwards and Letter-Number Sequencing-WAIS III or WISC IV), and inhibition (Color Word Interference-D-KEFS). None of these latter cognitive skills were specifically targeted during training, suggesting spill-over effects of the Gist training to untrained cognitive domains. The Memory trained group's performance on memory for facts approached significance; however, results failed to show significant gains in domains of abstracting meaning, working memory, inhibition, or other cognitive areas.

Taken together, evidence from these three studies implicates the potential to enhance cognitive performance in areas of cognitive control in typically developing teens and teens with TBI, beyond the traditional treatment phase of 3 months post injury. Augmenting cognitive performance in normally developing populations and individuals at chronic stages post brain injury represents newer and promising areas of investigation.

\section{ADULT COGNITIVE TRAINING TRIALS (ANAND ET AL., 2011; VAS ET AL., 2011; CHAPMAN ET AL., 2013) Adults with TBI}

In a randomized trial, the effects of Gist-reasoning training were compared to an active control training involving New learning in adults with TBI (ages 29-65 years) in chronic stages post-injury (>one year) (Vas et al., 2011). The Gist-trained group exhibited significant gains in abstracting meanings from complex information as compared to the New learning training group. Moreover, the Gist-trained group showed significant enhancement on measures of immediate memory (Digit Span Forward-WAIS-III), executive functions of working memory (Letter Number-Sequence-WAIS III and Daneman and Carpenter listening span) and cognitive switching (Color-Word Interference task-D-KEFS), and non-verbal reasoning (Matrix Reasoning-WAIS III). None of these latter cognitive processes were specifically targeted during training, adding further evidence of generalized benefits from Gist training. Furthermore, this group reported significant improvement in daily life skills (GOS-E, Functional Status Examination, Community Integration Questionnaire), such as increased socialization (e.g., initiating and planning activities with family and friends), higher levels of life productivity (e.g., active job seeking, setting up interviews, improved work efficiency and output), improved personal management (e.g., completing household responsibilities and house upkeep) and better sense of overall well-being. These reported real life gains from Gist training are not likely due to placebo effects since both groups believed they were receiving the experimental training. These training-related gains were maintained at 6-month follow-up. Despite comparable levels of active and engaged learning, the New Learning group failed to show significant gains on any of the performance measures.

\section{Adults with pre-clinical Alzheimer's (Mild Cognitive Impairment)}

This study compared immediate benefits of Gist training vs. New learning on cognition in individuals with Mild Cognitive Impairment (MCI) in a random assignment design (Mudar et al., 2013). Groups were comparable in age, Mini-Mental State Examination (MMSE) and episodic memory scores at baseline. Both groups received $8 \mathrm{~h}$ of training over a period of 4 weeks. We found differential cognitive gains between the two groups. Significant improvement was observed in the Gist-trained group on measures of abstract meaning, strategic attention, memory (immediate and delayed recall Logical Memory subtest-WMS III) and abstract verbal reasoning (Similarities-WAIS III). In contrast, participants in the New learning group showed significant improvements in remembering facts on an experimental text memory measure and on the Sorting test (D-KEFS). These findings suggest that both trainings offered some benefit for those with a progressive neurologic disease; however, Gist training offered broader benefits. Given the lack of pharmacological treatment options in slowing cognitive deterioration in pre-clinical stages of dementia, one current focus is on identifying nonpharmacological options (e.g., cognitive training) that can slow the rate of cognitive deterioration. Although maintenance of gains from Gist training needs to be studied in larger trials, even shortterm increases in cognitive performance offer a glimmer of hope. Different doses and more frequent time intervals of cognitive training needs to be examined to harness optimal benefits in terms of maintaining cognitive capacity and slowing decline.

\section{Cognitively healthy adults}

The short-term effects of (i.e., 12 weeks of 1 -h in-person training/week $+2 \mathrm{~h}$ /week homework) Gist training on cognitive and neural plasticity was examined in cognitively healthy 

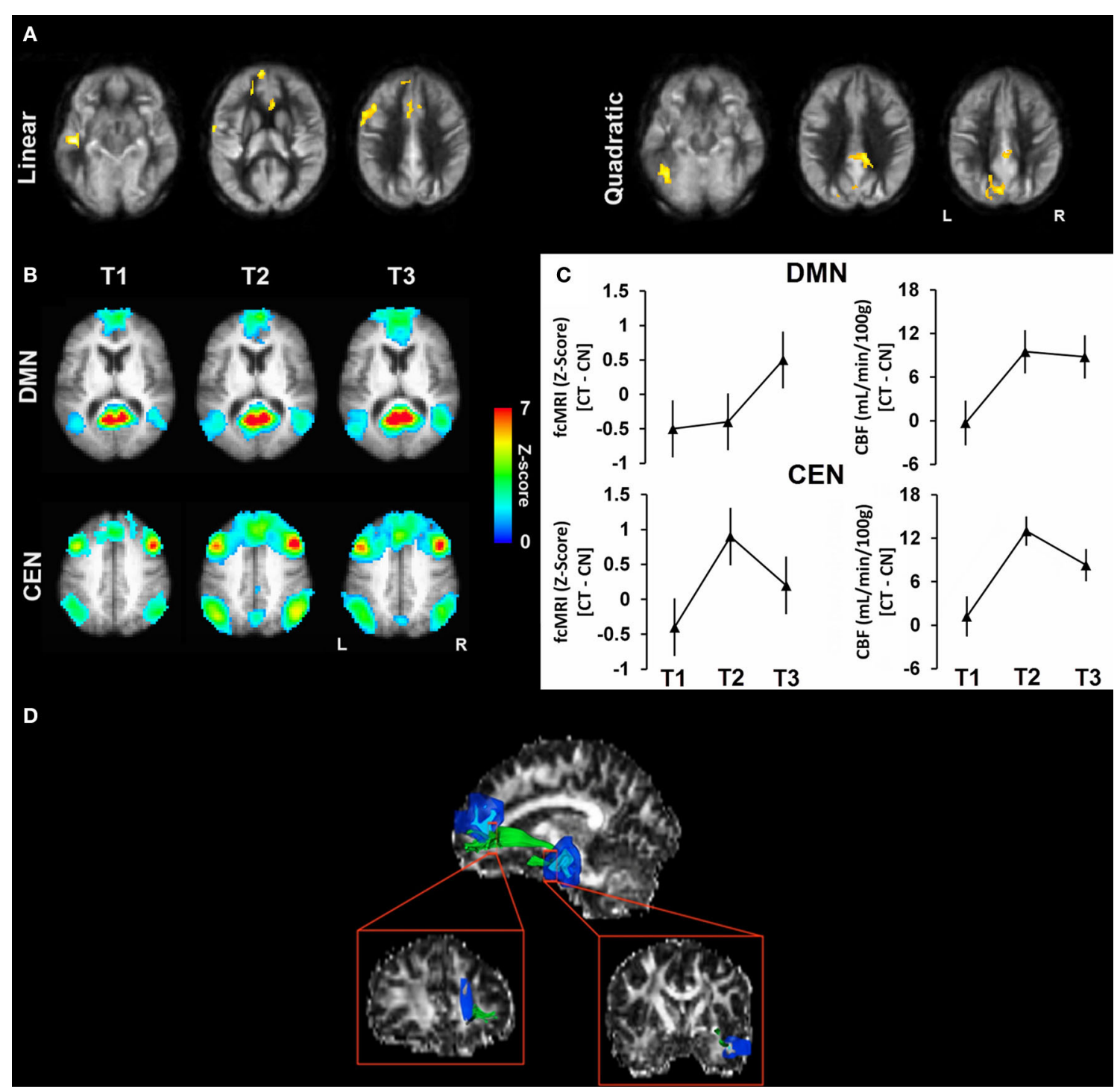

FIGURE 1 | This figure illustrates the convergence of neural plasticity findings in the cognitive training vs. control group across cerebral blood flow, functional connectivity, and structural DTI changes implicating functional brain changes more frequent and rapid than structural plasticity comparing changes at T2 and T3 to baseline T1 measures. (A) Results of CBF voxel-based comparison superimposed on an average CBF map of all participants for linear and quadratic interaction contrasts. (B) The average functional connectivity maps (i.e., DMN and CEN) of the cognitive training group are overlaid on their average T1-weighted image. (C) Mean increase in fcMRI z-scores (left column) and mean change in absolute CBF (right column) are shown for DMN and CEN across time periods. (D) A representative participant's uncinate fasciculus (green) is overlaid on his fractional anisotropy map from DTI (Chapman et al., 2013). adults (55-75 years of age), well-screened to rule out early dementia, as compared to a Wait-list control group (Chapman et al., 2013). Individuals were assessed at baseline, at midpoint and immediately post-training. The Gist-trained group showed generalized cognitive gains consistent with an earlier pilot study in healthy older adults in which participants improved in cognitive switching (Trail Making Test Part B), concept abstraction (Similarities-WAIS III), and fluency (COWAT letter fluency) (Anand et al., 2011). We examined changes in large-scale brain networks in terms of brain blood flow (CBF) and functional and structural connectivity, in addition to the relationship between improved cognitive performance and brain changes. Results revealed significant increases in global CBF (total brain) as well as increased regional CBF measures as measured with Arterial Spin Labeling in two distinct and major brain networks tied to higher-order cognition, namely the Default Mode Network (DMN) and the Central Executive Network (CEN). Specifically, the DMN and CEN have been associated with top-down, cognitive control processes (Bressler and Menon, 2010). We also found corroborating patterns of increased functional connectivity in these very same major brain networks as the increased CBF (Figure 1). Additionally, we found significantly increased 
structural connectivity as measured with diffusion tensor imaging (DTI) in the left uncinate fasciculus-the white matter tract that connects the middle temporal lobe to the superior medial frontal gyri after 12 weeks of training. The finding of increased white matter integrity in this select brain region perhaps suggests that some degree of atrophy at the level of white matter tracts in healthy aging may be reversible through Gist training. The expansion of the uncinate is intriguing and perhaps suggests that this pathway plays a role in synthesizing new learninglinking the memory center (left middle temporal region) to a brain region that is implicated in abstraction skills (e.g., the left superior medial frontal gyrus).

Whereas a handful of studies have reported cognitive gains and brain changes in response to cognitive training (Nyberg et al., 2003; Boyke et al., 2008; Belleville et al., 2011; Rosen et al., 2011), this study provided some of the first convergent findings across multiple brain imaging platforms (CBF, connectivity, white matter) and cognition supporting the promise of strategy-based topdown cognitive training to enhance brain integrity (Chapman et al., 2013). We acknowledge these findings are preliminary and should be interpreted with caution since the gist training group was compared to a wait-list control group. Nonetheless, the significant relation between brain changes and cognitive improvement provides impetus that the brain gains from gist-training may be possible and warrant further study with active controls.

\section{CONCLUSIONS AND FUTURE OPPORTUNITIES}

This synopsis of key findings across studies in normal and clinical populations indicates Gist reasoning training has the potential to improve cognitive performance beyond skills trained with the likelihood of enhancing underlying neural systems, as well as real life functional abilities. The significant improvements were achieved after relatively short-term training periods. The gains were documented in pseudo-randomly assigned trials comparing the experimental Gist reasoning training to control groups using objective measures.

We postulate that top-down strategy-based cognitive training may yield efficient and easily adoptable methods of mental practice to achieve broad-based benefits. To guide future cognitive training trials, we offer several explanations why Gist reasoning training may augment higher-order executive functions. First, gist reasoning takes advantage of the human brain's preferential bias toward understanding generalized/gist meanings (Bartlett, 1932; Reyna, 1996; Gabrieli, 2004). Extant evidence has demonstrated that while memory for details is lost fairly quickly, memory for global/gist meanings is preserved when delayed recall is examined, whether tested $30 \mathrm{~min}$, one day or even a week later (Bransford and Franks, 1971; Bransford et al., 1972; Mandler and Rabinowitz, 1981; Reyna, 1996; Norman and Schacter, 1997; Kahana and Wingfield, 2000; Gabrieli, 2004). Second, gist reasoning requires an active process of meaningabstraction where the incoming details are integrated within one's repository of world knowledge by a conscious, controlled manipulation of input into précised ideas (Johnson-Laird, 1983; Van Dijk et al., 1983; Frederksen and Donin, 1991; Zwaan and Radvansky, 1998; Chapman and Mudar, 2013). This integration of incoming data with prior knowledge necessitates activation of top-down processing with enhanced depth of encoding compared to simple representation of literal input. Third, gist reasoning is a practical skill that the majority of people from adolescence to old age can implement and practice throughout their daily normal mental activities (Lloyd and Reyna, 2009; Gamino et al., 2010; Vas et al., 2011; Motes et al., 2014). Examples of gist reasoning are meaning-creations illustrated by generating interpretations, themes, take-home messages, synopses, or generalized statements, to mention a few forms. Fourth, accruing evidence suggests that such a top-down approach to processing engages broad-based brain networks (Gazzaley et al., 2005; Chen et al., 2006, 2011; Chapman et al., 2013). We propose that when the neural activity of major brain networks is increased through complex and meaningful cognitive activities involving gist reasoning, the outcomes may be manifested at multiple levels of cognitive performance and neural health.

Immense potential exists to augment cognitive performance and enhance neural systems through top-down cognitive activity, such as gist reasoning. Future research opportunities should combine multiple approaches simultaneously from the growing armamentaria shown to enhance cognitive performance and brain functions to examine additive benefits. The current reported benefits from Gist reasoning training motivate future trials where the gist training protocol is combined with methods such as short-term meditation (Tang et al., 2007), sensory processing training (Mahncke et al., 2006), aerobic exercise (Kramer and Erickson, 2007; Chapman et al., 2013), or protocols to improve sleep (Nedergaard, 2013). Also critical is examining subjects' motivational level as a factor. Expanded efforts to identify combinatorial protocols that strengthen cognitive performance and recoup losses will be of major public health significance, with the ultimate goal to more fully harness the brain's capacity to be strengthened in health, disease, and injury.

\section{FUNDING}

This work was supported by grant from the National Institute of Health (RC1-AG035954, R01-NS067015, R01AG033106, NICHD R21-HD062835), Department of Defense (W81XWH-11-2-0194) and by grants from the T. Boone Pickens Foundation, the RGK foundation, the Lyda Hill Foundation, Dallas Foundation, and Dee Wyly Distinguished University Endowment.

\section{ACKNOWLEDGMENTS}

The authors thank Jacquelyn Gamino, Lori Cook, Asha Vas, Mike Motes, Molly Keebler, Audette Rackley, Jennifer Zientz, Erin Venza, Hsueh-Sheng Chiang, Justin Eroh, Ali Perez, and the rest of the cognitive training team for their contribution to the research discussed in this perspective.

\section{REFERENCES}

Anand, R., Chapman, S. B., Rackley, A., Keebler, M., Zientz, J., and Hart, J. (2011). Gist reasoning training in cognitively normal seniors. Int. J. Geriatr. Psychiatry 26, 961-968. doi: 10.1002/gps.2633

Anguera, J. A., Boccanfuso, J., Rintoul, J. L., Al-Hashimi, O., Faraji, F., Janowich, J., et al. (2013). Video game training enhances cognitive control in older adults. Nature 501, 97-101. doi: 10.1038/nature12486

Bartlett, F. C. (1932). Remembering: An Experimental and Social Study. Cambridge: Cambridge University. 
Belleville, S., Clément, F., Mellah, S., Gilbert, B., Fontaine, F., and Gauthier, S. (2011). Training-related brain plasticity in subjects at risk of developing Alzheimer's disease. Brain 134, 1623-1634. doi: 10.1093/brain/awr037

Bennett, E. L., Diamond, M. C., Krech, D., and Rosenzweig, M. R. (1964). Chemical and Anatomical Plasticity of the Brain. Science 610-619. doi: 10.1126/science.146.3644.610

Binder, D. T. G., O'Connor, C., Levine, B. (2008). Brain HealthWorkshop. Rotman Research Institute, Baycrest, Toronto: ON; University of California, Berkeley: CA.

Boyke, J., Driemeyer, J., Gaser, C., Büchel, C., and May, A. (2008). Traininginduced brain structure changes in the elderly. J. Neurosci. 28, 7031-7035. doi: 10.1523/JNEUROSCI.0742-08.2008

Bransford, J. D., Barclay, J. R., and Franks, J. J. (1972). Sentence memory: a constructive versus interpretive approach. Cogn. Psychol. 3, 193-209. doi: 10.1016/0010-0285(72)90003-5

Bransford, J. D., and Franks, J. J. (1971). The abstraction of linguistic ideas. Cogn. Psychol. 2, 331-350. doi: 10.1016/0010-0285(71)90019-3

Bressler, S. L., and Menon, V. (2010). Large-scale brain networks in cognition: emerging methods and principles. Trends Cogn. Sci. 14, 277-290. doi: 10.1016/j.tics.2010.04.004

Carlson, M. C., Erickson, K. I., Kramer, A. F., Voss, M. W., Bolea, N., Mielke, M., et al. (2009). Evidence for neurocognitive plasticity in at-risk older adults: the experience corps program. J. Gerontol. A Biol. Sci. Med. Sci. 64, 1275-1282. doi: 10.1093/gerona/glp117

Chapman, S. B., Aslan, S., Spence, J. S., Hart, J. J., Bartz, E. K., Didehbani, N., et al. (2013). Neural mechanisms of brain plasticity with complex cognitive training in healthy seniors. Cereb. Cortex. doi: 10.1093/cercor/bht234. [Epub ahead of print].

Chapman, S. B., Gamino, J. F., and Mudar, R. A. (2012). "Higher-order strategic gist reasoning in adolescence," in The Adolescent Brain: Learning, Reasoning, and Decison Making, eds V. Reyna, S. B. Chapman, M. R. Dougherty, and J. Confrey, 123-151. doi: 10.1037/13493-005

Chapman, S. B., and Mudar, R. A. (2013). Discourse gist: a window into the brain's complex cognitive capacity. Discourse Stud. 15, 519-533. doi: $10.1177 / 1461445613501444$

Chen, A. J., Nycum, T. J., Novakovic-Agopian, T., Song, S., Gary, T., Rome, S., et al. (2011). Training of goal-directed attention regulation enhances control over neural processing for individuals with brain injury. Brain 134, 1541-1554. doi: 10.1093/brain/awr067

Chen, A. J.-W., Abrams, G. M., and D'Esposito, M. (2006). Functional reintegration of prefrontal neural networks for enhancing recovery after brain injury. J. Head Trauma Rehabil. 21, 107-118. doi: 10.1097/00001199-20060300000004

Clare, L., and Woods, R. T. (2004). Cognitive training and cognitive rehabilitation for people with early-stage Alzheimer's disease: a review. Neuropsychol. Rehabil. 14, 385-401. doi: 10.1080/09602010443000074

Dahlin, E., Neely, A. S., Larsson, A., Bäckman, L., and Nyberg, L. (2008). Transfer of learning after updating training mediated by the striatum. Science 320 1510-1512. doi: 10.1126/science.1155466

Frederksen, C. H., and Donin, J. (1991). Constructing multiple semantic representations in comprehending and producing discourse. Adv. Psychol. 79, 19-44. doi: 10.1016/S0166-4115(08)61547-2

Gabrieli, J. (2004). Memory: pandora's hippocampus? Cerebrum 6, 39-48.

Gamino, J. F., Chapman, S. B., Hull, E. L., and Lyon, G. R. (2010). Effects of higher-order cognitive strategy training on gist-reasoning and fact-learning in adolescents. Front. Psychol. 1:188. doi: 10.3389/fpsyg.2010.00188

Gates, N. J., Sachdev, P. S., Fiatarone Singh, M. A., and Valenzuela, M. (2011). Cognitive and memory training in adults at risk of dementia: a systematic review. BMC Geriatr. 11:55. doi: 10.1186/1471-2318-11-55

Gazzaley, A., Cooney, J. W., McEvoy, K., Knight, R. T., and D'Esposito, M. (2005). Top-down enhancement and suppression of the magnitude and speed of neural activity. J. Cogn. Neurosci. 17, 507-517. doi: 10.1162/0898929053 279522

Jaeggi, S. M., Buschkuehl, M., Jonides, J., and Perrig, W. J. (2008). Improving fluid intelligence with training on working memory. Proc. Natl. Acad. Sci. U.S.A. 105 6829-6833. doi: 10.1073/pnas.0801268105

Johnson-Laird, P. N. (1983). Mental Models: Towards a Cognitive Science of Language, Inference, and Consciousness. Cambridge, MA: Harvard University Press.
Kahana, M. J., and Wingfield, A. (2000). A functional relation between learning and organization in free recall. Psychon. Bull. Rev. 7, 516-521. doi: 10.3758/BF03214365

Kramer, A. F., and Erickson, K. I. (2007). Capitalizing on cortical plasticity: influence of physical activity on cognition and brain function. Trends Cogn. Sci. 11, 342-348. doi: 10.1016/j.tics.2007.06.009

Lloyd, F. J., and Reyna, V. F. (2009). Clinical gist and medical education. JAMA 302, 1332-1333. doi: 10.1001/jama.2009.1383

Mahncke, H. W., Connor, B. B., Appelman, J., Ahsanuddin, O. N., Hardy, J. L., Wood, R. A., et al. (2006). Memory enhancement in healthy older adults using a brain plasticity-based training program: a randomized, controlled study. Proc. Natl. Acad. Sci. U.S.A. 103, 12523-12528. doi: 10.1073/pnas.0605194103

Mandler, G., and Rabinowitz, J. C. (1981). Appearance and reality: does a recognition test really improve subsequent recall and recognition? J. Exp. Psychol. Hum. Learn. Mem. 7, 79. doi: 10.1037/0278-7393.7.2.79

Martin, M., Clare, L., Altgassen, A. M., Cameron, M. H., and Zehnder, F. (2011). Cognition-based interventions for healthy older people and people with mild cognitive impairment. Cochrane Database Syst. Rev. CD006220. doi: 10.1002/14651858.CD006220.pub2

Motes, M. A., Gamino, J. F., Chapman, S. B., Rao, N. K., Maguire, M. J., Brier, M. R., et al. (2014). Inhibitory control gains from higher-order cognitive strategy training. Brain Cogn. 84, 44-62. doi: 10.1016/j.bandc.2013.10.007

Mudar, R. A., Chiang, H., Eroh, J. T., Rackley, A., Venza, E., Martin-Cook, K., et al. (2013). "Benefits of cognitive training in individuals with mild cognitive impairment," in Poster Presentation - Cognitive Neuroscience Society Annual Meeting, April 2013 (San Francisco, CA)

Nedergaard, M. (2013). Garbage truck of the brain. Science 340, 1529-1530. doi: $10.1126 /$ science. 1240514

Norman, K. A., and Schacter, D. L. (1997). False recognition in younger and older adults: exploring the characteristics of illusory memories. Mem. Cognit. 25, 838-848. doi: 10.3758/BF03211328

Nyberg, L., Sandblom, J., Jones, S., Neely, A. S., Petersson, K. M., Ingvar, M., et al. (2003). Neural correlates of training-related memory improvement in adulthood and aging. Proc. Natl. Acad. Sci. U.S.A. 100, 13728-13733. doi: 10.1073/pnas. 1735487100

Papp, K. V., Walsh, S. J., and Snyder, P. J. (2009). Immediate and delayed effects of cognitive interventions in healthy elderly: a review of current literature and future directions. Alzheimers Dement. 5, 50-60. doi: 10.1016/j.jalz.2008. 10.008

Rebok, G. W., Ball, K., Guey, L. T., Jones, R. N., Kim, H. Y., King, J. W., et al. (2014) Ten year effects of the advanced cognitive training for independent and vital elderly cognitive training trial on cognition and everyday functioning in older adults. J. Am. Geriatr. Soc. 62, 16-24. doi: 10.1111/jgs.12607

Reijnders, J., Van Heugten, C., and Van Boxtel, M. (2012). Cognitive interventions in healthy older adults and people with mild cognitive impairment; a systematic review. Ageing Res. Rev. 12, 263-275. doi: 10.1016/j.arr.2012.07.003

Reyna, V. F. (1996). Conceptions of memory development with implications for reasoning and decision making. Ann. Child Dev. 12, 87-118.

Reyna, V. F., and Brainerd, C. J. (1995). Fuzzy-trace theory: an interim synthesis. Learn. Individ. Differ. 7, 1-75. doi: 10.1016/1041-6080(95)90031-4

Reyna, V. F., and Brainerd, C. J. (2011). Dual processes in decision making and developmental neuroscience: a Fuzzy-Trace model. Dev. Rev. 31, 180-206. doi: 10.1016/j.dr.2011.07.004

Reyna, V. F., and Lloyd, F. J. (2006). Physician decision making and cardiac risk: effects of knowledge, risk perception, risk tolerance, and fuzzy processing. J. Exp. Psychol. Appl. 12, 179-195. doi: 10.1037/1076-898X.12.3.179

Rosen, A. C., Sugiura, L., Kramer, J. H., Whitfield-Gabrieli, S., and Gabrieli, J. D. (2011). Cognitive training changes hippocampal function in mild cognitive impairment: a pilot study. J. Alzheimers Dis. 26, 349-357. doi: 10.3233/JAD2011-0009

Schooler, C. (1984). Psychological effects of complex environments during the life span: a review and theory. Intelligence 8, 259-281. doi: 10.1016/01602896(84)90011-4

Schooler, C., Mulatu, M. S., and Oates, G. (1999). The continuing effects of substantively complex work on the intellectual functioning of older workers. Psychol. Aging 14, 483. doi: 10.1037/0882-7974.14.3.483

Tang, Y.-Y., Ma, Y., Wang, J., Fan, Y., Feng, S., Lu, Q., et al. (2007). Short-term meditation training improves attention and self-regulation. Proc. Natl. Acad. Sci. U.S.A. 104, 17152-17156. doi: 10.1073/pnas.0707678104 
Tardif, S., and Simard, M. (2011). Cognitive stimulation programs in healthy elderly: a review. Int. J. Alzheimers Dis. 2011:378934. doi: 10.4061/2011/378934

Teixeira, C. V. L., Gobbi, L. T. B., Corazza, D. I., Stella, F., Costa, J. L. R., and Gobbi, S. (2012). Non-pharmacological interventions on cognitive functions in older people with mild cognitive impairment (MCI). Arch. Gerontol. Geriatr. 54, 175-180. doi: 10.1016/j.archger.2011.02.014

Thompson, T. W., Waskom, M. L., Garel, K.-L. A., Cardenas-Iniguez, C., Reynolds, G. O., Winter, R., et al. (2013). Failure of working memory training to enhance cognition or intelligence. PLoS ONE 8:e63614. doi: 10.1371/journal.pone.0063614

Valenzuela, M. J., Sachdev, P., Wen, W., Chen, X., and Brodaty, H. (2008). Lifespan mental activity predicts diminished rate of hippocampal atrophy. PLoS ONE 3:e2598. doi: 10.1371/journal.pone.0002598

Valenzuela, M., and Sachdev, P. (2009). Can cognitive exercise prevent the onset of dementia? Systematic review of randomized clinical trials with longitudinal follow-up. Am. J. Geriatr. Psych. 17, 179-187. doi: 10.1097/JGP.0b013e3181953b57

Van Dijk, T. A., Kintsch, W., and Van Dijk, T. A. (1983). Strategies of Discourse Comprehension. New York, NY: Academic Press.

Vas, A. K., Chapman, S. B., Cook, L. G., Elliott, A. C., and Keebler, M. (2011). Higher-order reasoning training years after traumatic brain injury in adults. J. Head Trauma Rehabil. 26, 224-239. doi: 10.1097/HTR.0b013e318 $218 \mathrm{dd} 3 \mathrm{~d}$
Willis, S. L., Tennstedt, S. L., Marsiske, M., Ball, K., Elias, J., Koepke, K. M., et al. (2006). Long-term effects of cognitive training on everyday functional outcomes in older adults. JAMA 296, 2805-2814. doi: 10.1001/jama.296.23.2805

Zelinski, E. M. (2009). Far transfer in cognitive training of older adults. Restor. Neurol. Neurosci. 27, 455-471. doi: 10.3233/RNN-2009-0495

Zwaan, R. A., and Radvansky, G. A. (1998). Situation models in language comprehension and memory. Psychol. Bull. 123:162. doi: 10.1037/0033-2909.123.2.162

Conflict of Interest Statement: The authors declare that the research was conducted in the absence of any commercial or financial relationships that could be construed as a potential conflict of interest.

Received: 31 January 2014; accepted: 10 April 2014; published online: 28 April 2014. Citation: Chapman SB and Mudar RA (2014) Enhancement of cognitive and neural functions through complex reasoning training: evidence from normal and clinical populations. Front. Syst. Neurosci. 8:69. doi: 10.3389/fnsys.2014.00069

This article was submitted to the journal Frontiers in Systems Neuroscience.

Copyright (c) 2014 Chapman and Mudar. This is an open-access article distributed under the terms of the Creative Commons Attribution License (CC BY). The use, distribution or reproduction in other forums is permitted, provided the original author(s) or licensor are credited and that the original publication in this journal is cited, in accordance with accepted academic practice. No use, distribution or reproduction is permitted which does not comply with these terms. 